\title{
The candidate filament close to the 3C 295 galaxy cluster: optical and X-ray spectroscopy ${ }^{\star}$
}

\author{
V. D’Elia ${ }^{1}$, F. Fiore ${ }^{1}$, S. Mathur ${ }^{2}$, and F. Cocchia ${ }^{1}$ \\ 1 INAF - Osservatorio Astronomico di Roma via Frascati 33, Monteporzio-Catone (RM) I00040, Italy \\ e-mail: delia@mporzio.astro.it \\ 2 The Ohio State University Columbus, OH 43210, USA
}

Received 28 December 2007 / Accepted 29 February 2008

ABSTRACT

\begin{abstract}
We present a detailed analysis of the overdensity of X-ray sources close to the $3 \mathrm{C} 295$ galaxy cluster $(z=0.46)$ to assess whether it is associated with a filament of the large-scale structure of the Universe. We obtained optical spectra of the optical counterparts of eleven sources associated with the filament, finding that one is at $z=0.474$. This is a type 1 AGN at 1.5 arcmin from the cluster center. We found three more sources with a redshift in the range $0.37 \div 0.53$. We extracted the stacked X-ray spectrum of $47 \mathrm{X}$-ray sources belonging to the putative filament. We found a significant narrow (at the resolution of the Chandra ACIS-I) line at $E \sim 4.4 \mathrm{keV}$, the energy of the iron $\mathrm{K} \alpha$ line at the redshift of the cluster. The detection of this line is confirmed at a confidence level of better than $3 \sigma$ and its energy is constrained to be in the range 6.2-6.47 (at a 90\% confidence level), excluding an identification with the 6.7 helium-like iron line from the hot cluster ICM at better than $4 \sigma$. We conclude that the detection of the redshifted line is a strong indication that at least several of the excess sources lie at $z \sim 0.46$ and that AGNs are efficient tracers of the "filament" connected with the central cluster of galaxies.
\end{abstract}

Key words. X-rays: galaxies - galaxies: clusters: individual: 3C 295 - X-rays: diffuse background cosmology: large-scale structure of Universe

\section{Introduction}

$N$-body and hydrodynamical simulations show that clusters of galaxies lie at the nexus of several filaments of galaxies (see e.g. Peacock 1999; Davè et al. 2001, and references therein). Such a "cosmic web" of voids and filaments maps out the largescale structure (LSS) of the Universe. According to the same simulations, these filaments contain a large fraction (30-40\%) of the baryons in the Universe at $z<1$; the remainder end up in the hot gas in clusters of galaxies, and in stars and cold gas clouds. Despite its larger total mass, observations of the intergalactic matter in filaments have yielded only limited information, mostly due to its low density: most of the baryons in this phase should be at densities only 10-100 times higher than the average density of the Universe. The most direct way to detect a filament at low redshift is by means of its soft X-ray diffuse emission (see e.g. Zappacosta et al. 2002; Soltan et al. 2002), or using soft X-ray and UV absorption-line studies (see e.g. Fiore et al. 2000, and references therein; Nicastro et al. 2002, 2003; Mathur et al. 2003; Nicastro 2005a,b). Both methods require very difficult observations, at the limit of the present generation of X-ray and UV facilities.

Alternatively, filaments could be mapped out by galaxies (Daddi et al. 2001; Giavalisco \& Dickinson 2001) and by the much more luminous Active Galactic Nuclei (AGNs), assuming that AGNs trace galaxies. Since rich clusters of galaxies are good indicators of regions of sky where filaments converge, numerous AGN searches around clusters of galaxies have been performed

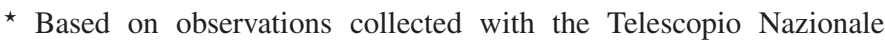
Galileo, Roque de Los Muchachos, La Palma, TF - Spain, and with the Chandra X-ray observatory. in the past. Several of these studies suggest that overdensities of AGNs exist around distant clusters of galaxies (Molnar et al. 2002, for the cluster Abell 1995; Best et al. 2002, for MS105403; Martini et al. 2002, for Abell 2104; Pentericci et al. 2002, for the protocluster at $z \sim 2.16$ around the radio galaxy MRC 1138206; see also Almaini et al. 2003, for the ELAIS North field). Cappelluti et al. (2005) performed the first systematic study of serendipitous X-ray source density around 10 high- $z$ clusters; they found a factor of approximately two overdensity in 4 cluster fields. Many of these studies have been performed in X-rays, since extragalactic X-ray sources, which are mostly AGNs, have a space density $\sim 10$ times higher than opticallyselected AGNs (see Yang et al. 2003), and therefore provide denser tracing of LSS. Later studies suggested that AGNs are more frequently found close to rich clusters than previously thought. Ruderman \& Ebeling (2005) studied the spatial distribution of a $95 \%$ complete sample of 508 X-ray point sources detected in the $0.5-2 \mathrm{keV}$ band by Chandra ACIS-I close to 51 massive galaxy clusters $(z \sim 0.3 \div 0.7)$. They found a significant $(8 \sigma)$ excess within $3.5 \mathrm{Mpc}$ of the cluster center, that they attribute to AGNs connected to the central clusters. Similarly, Martini et al. (2006) spectroscopically-identified 40 sources in the field of 8 Chandra low-redshift clusters $(0.05<z<0.31)$. Using a combination of optical emission lines, X-ray spectral shapes, and multiwavelength flux ratios, they concluded that at least 35 of these objects have AGN signatures. Dilution of lowluminosity AGN spectral signatures by host-galaxy starlight and obscuration of accretion onto the central black hole do not easily allow sources to be optically classified as AGNs. Even though they refer to cluster members, we stress that the spectroscopic identification of sources close to clusters cannot be an easy task. 
In this paper, we study the X-ray source excess close to the 3C 295 galaxy cluster. Cappi et al. (2001) observed the Chandra $8 \times 8$ arcmin ACIS field around 3C $295(z=0.46)$. They reported the tentative $0.5-2 \mathrm{keV}$ detection of an overdensity of faint X-ray sources in a region of a few arcmin around the cluster, with respect to the average X-ray source density at the same flux limit. However, the observation was too short $(\sim 18 \mathrm{ks})$, and the source samples consequently too small, to derive any more detailed conclusion. D'Elia et al. (2005) observed the 3C 295 field using Chandra for about $100 \mathrm{ks}$. They performed a detailed study of the field, applying three different methods of analysis, namely, the $\log N-\log S$ computed separately for each of the four ACIS-I chips, the two point, two-dimensional KolmogorovSmirnov (KS) test, and the angular correlation function of the field. All of these analysis methods suggest an asymmetric distribution of sources in the NE region of the field and a strong clustering on scales of a few arcmins.

The aim of this work is to investigate whether the asymmetric distribution of X-ray sources close to the 3C 295 galaxy cluster, found by D'Elia et al. (2005), is associated with a "filament" of the LSS of the Universe and connected to the central cluster. The paper is organized as follows: Sect. 2 presents the optical identifications of the X-ray Chandra sources; Sect. 3 presents the analysis of the X-ray stacked spectrum of the sources in the overdensity region; Sect. 4 discusses the results and draws our conclusions. We adopt in the following a concordance cosmology with $H_{0}=65 \mathrm{~km} \mathrm{~s}^{-1} \mathrm{Mpc}^{-1}, \Omega_{\mathrm{M}}=0.3$ and $\Omega_{\Lambda}=0.7$.

\section{Optical analysis}

The asymmetric distribution of the sources around the 3C 295 galaxy cluster and the overdensity in the NE region of the 3C 295 field found by D'Elia et al. (2005) posed the obvious question of whether such overdensities are due to background or foreground objects, or if the excess sources are spatially linked to the central cluster along a filmament of the LSS of the Universe. The natural way to answer this question is to optically identify the X-ray sources and determine their redshift by means of an analysis of the spectrum.

\subsection{Data acquisition and reduction}

To acquire the optical spectra of the 3C 295 sources, we proposed a Multi Object Spectroscopy (MOS) programme using DOLORES@TNG (AOT 9). The project was accepted and two nights of observation were allocated. Two acquisition images each of exposure time $900 \mathrm{~s}$ and with a limiting magnitude of $R \sim 24$, had already been obtained using DOLORES on March 19, 2002. These images were centered on the NE and SW quadrants of the Chandra field, and covered an area of $9^{\prime} \times 9^{\prime}$, with respect to the $16^{\prime} \times 16^{\prime}$ area of the four ACIS-I chips. We acquired the spectral data on 22 and 23 March, 2004. We positioned in slit 26 optically-identified X-ray sources whose optical counterpart was brighter than $R=22$. Unfortunately, due to weather and technical difficulties, we were able to observe our target field for only six hours.

All spectra acquired were collected into three MOS images. The data were reduced using the IRAF software and its standard analysis packages. Each MOS image was corrected for the electronic bias; the spectra were then separated, flatfielded and background-subtracted. Finally, we removed cosmic rays and performed a wavelength calibration for each spectrum.

\subsection{Spectral analysis and redshift determination}

Table 1 summarizes the results of our optical identification campaign. In detail, we show the X-ray and optical coordinates, the $R$ magnitude, and the $0.5-10 \mathrm{keV} \mathrm{X}$-ray flux. The identification number given in the first column refers to the whole $0.5-10 \mathrm{keV}$ sample in D'Elia et al. (2005). Column 8 indicates the Chandra ACIS-I chip to which each X-ray source belongs, while Col. 9 indicates the sources selected for the stacked X-ray spectrum, as described in the next section.

We were able to obtain reliable redshifts for only 11 of the sources placed inside the slit. This is because the optical counterparts of several X-ray objects were too faint for a reasonable signal-to-noise ratio to be achieved in the observing time and seeing conditions. The last three columns of Table 1 report the redshift, the X-ray (0.5-10 keV) luminosity, and the classification of the spectroscopically-identified sources.

Figure 1 shows the position of these sources inside the 3C 295 field. Three of the 11 sources were found to be stars, and 2 of these are located in the SW part of the 3C 295 field. This enhances the asymmetric distribution found by D'Elia et al. (2005), who showed that the SW region was underpopulated, with only 12 X-ray sources identified in the $0.5-10 \mathrm{keV}$ band of a total of 121 sources in the entire field. The star identified in the NE chip cannot be the true optical counterpart of the X-ray source, because a fainter source, inaccessible to $4 \mathrm{~m}$ telescope spectroscopy, lies within the X-ray error box; a question mark identifies this source in Fig. 1.

One of the sources in the NE region has a redshift of 0.474 (see Fig. 2 upper panel). This is a type $1 \mathrm{AGN}$ at $1.5 \mathrm{arcmin}$ from the center of the cluster, implying a physical distance of $600 \mathrm{kpc}$. The spread in velocity with respect to the cluster redshift is $4200 \mathrm{~km} \mathrm{~s}^{-1}$. Three more sources have a redshift in the range $0.37 \div 0.53$ : two of these are located in the NE region, while one is in the SW region. Their spectra are shown in Fig. 2. The remaining sources are two $z>1$ QSOs and two lower redshift galaxies $(z<0.3)$.

The identification of only 1 source at the redshift of 3C 295 is unsurprising. D'Elia et al. (2005) showed that the $0.5-10 \mathrm{keV}$ luminosity of the sources in the 3C 295 field should be in the range $7.5 \times 10^{41} \div 10^{44} \mathrm{erg} \mathrm{s}^{-1}$ if they were associated to filaments connected to the central cluster of galaxies. Therefore several starburst galaxies and low luminosity AGNs are expected in this structure. Assuming a typical X-ray to optical flux ratio (e.g. Fiore et al. 2003), one would expect that their optical magnitude would be fainter than $R=22$. These magnitudes are beyond the reach of our $4 \mathrm{~m}$-class telescopes. Our spectroscopic run was able to identify only optically unobscured QSOs and bright Seyfert galaxies at $z \sim 0.46$. At similar redshift, the identification of sources that are both optically fainter and highlyobscured will require data from $8 \mathrm{~m}$-class telescopes.

Fortunately, optical spectroscopy is not the only tool available to search for evidence of the association of the X-ray sources with the large-scale structure linked to 3C 295. In the next section, we perform a new test based on X-ray spectroscopy.

\section{X-ray analysis}

We search for more hints to support the presence of a filament spatially-connected to the 3C 295 cluster, using Chandra data. Due to the sharpness of the Chandra Point Spread Function, background counts at the position of the X-ray sources are often negligible. Therefore, "stacking" together spectra extracted at the position of each source almost increases linearly both 
Table 1. Optical identification of the x-ray sources in the 3C 295 field.

\begin{tabular}{|c|c|c|c|c|c|c|c|c|c|c|c|}
\hline & \multicolumn{2}{|c|}{$\bar{X}$ X position } & \multicolumn{2}{|c|}{ "Optical position } & \multicolumn{2}{|r|}{ 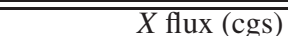 } & & & & & \\
\hline$n$ & $\overline{R A}$ & Dec & RA & Dec & $R$ mag & $0.5-10 \mathrm{keV}$ & Chip & Stack & $z$ & LX (erg/s) & Class \\
\hline 2 & $14: 10: 17.329$ & $52: 14: 39.7$ & $14: 10: 17.49$ & $52: 14: 38.55$ & 24.43 & $1.60 \mathrm{E}-15$ & 2 & $\mathrm{n}$ & - & - & \\
\hline 3 & $14: 10: 18.901$ & $52: 13: 13.98$ & 14:10:19.17 & 52:13:14.99 & 18.77 & $1.90 \mathrm{E}-15$ & 2 & $\mathrm{n}$ & - & - & \\
\hline 4 & $14: 10: 24.356$ & 52:9: 28.65 & $14: 10: 24.52$ & $52: 9: 27.53$ & 16.6 & $3.98 \mathrm{E}-15$ & 2 & $\mathrm{n}$ & 0 & - & Star \\
\hline 5 & $14: 10: 28.38$ & $52: 11: 32.56$ & $14: 10: 28.58$ & $52: 11: 33.25$ & 21.06 & $2.34 \mathrm{E}-15$ & 2 & $\mathrm{y}$ & - & - & \\
\hline 6 & $14: 10: 30.608$ & $52: 7: 34.86$ & $14: 10: 30.91$ & $52: 7: 35.8$ & 17.77 & $2.94 \mathrm{E}-15$ & 3 & $\mathrm{n}$ & 0 & - & Star \\
\hline 7 & $14: 10: 31.096$ & $52: 15: 33.3$ & $14: 10: 31.33$ & $52: 15: 33.9$ & 20.32 & $4.54 \mathrm{E}-14$ & 2 & $\mathrm{y}$ & - & - & \\
\hline 8 & $14: 10: 32.098$ & $52: 7: 45.19$ & $14: 10: 32.38$ & $52: 7: 45.74$ & 23.62 & $8.84 \mathrm{E}-15$ & 3 & $\mathrm{n}$ & - & - & \\
\hline 9 & $14: 10: 33.891$ & $52: 12: 11.35$ & $14: 10: 34.15$ & $52: 12: 11.6$ & 23.6 & $2.59 \mathrm{E}-15$ & 2 & $\mathrm{n}$ & - & - & \\
\hline 11 & $14: 10: 37.911$ & $52: 10: 44.4$ & $14: 10: 38.15$ & $52: 10: 44.87$ & 21.91 & $4.21 \mathrm{E}-15$ & 2 & $\mathrm{n}$ & - & - & \\
\hline 13 & $14: 10: 39.906$ & 52:13:7.61 & 14:10:39.91 & 52:13:8.99 & 24.2 & $1.02 \mathrm{E}-15$ & 2 & $\mathrm{y}$ & - & - & \\
\hline 14 & $14: 10: 40.287$ & $52: 11: 51.43$ & $14: 10: 40.56$ & $52: 11: 52.16$ & 23.5 & $9.04 \mathrm{E}-16$ & 2 & $\mathrm{n}$ & - & - & \\
\hline 16 & $14: 10: 41.578$ & $52: 7: 7.35$ & 14:10:41.59 & $52: 7: 5.62$ & 23.55 & $8.60 \mathrm{E}-16$ & 3 & $\mathrm{n}$ & - & - & \\
\hline 17 & $14: 10: 42.614$ & $52: 12: 57.53$ & $14: 10: 42.55$ & $52: 12: 57.11$ & 25.1 & $6.32 \mathrm{E}-16$ & 2 & $\mathrm{n}$ & - & - & \\
\hline 20 & $14: 10: 44.417$ & $52: 11: 59.43$ & $14: 10: 44.63$ & 52:12:0.07 & 21.94 & $5.42 \mathrm{E}-16$ & 2 & $\mathrm{n}$ & - & - & \\
\hline 21 & $14: 10: 45.979$ & $52: 7: 37.84$ & $14: 10: 45.98$ & $52: 7: 40.67$ & 21.66 & $2.05 \mathrm{E}-15$ & 3 & $\mathrm{n}$ & - & - & \\
\hline 23 & $14: 10: 49.855$ & $52: 11: 40.85$ & $14: 10: 49.63$ & $52: 11: 38.53$ & 23.35 & $5.98 \mathrm{E}-15$ & 2 & $\mathrm{y}$ & - & - & \\
\hline 25 & $14: 10: 52.372$ & $52: 9: 1.76$ & $14: 10: 52.68$ & $52: 9: 1.61$ & 23.91 & $6.46 \mathrm{E}-16$ & 3 & $\mathrm{n}$ & - & - & \\
\hline 28 & $14: 10: 55.898$ & $52: 13: 26.4$ & $14: 10: 56.23$ & $52: 13: 26.15$ & 23.89 & $4.34 \mathrm{E}-16$ & 2 & $\mathrm{y}$ & - & - & \\
\hline 29 & $14: 10: 56.927$ & $52: 12: 37.4$ & $14: 10: 56.96$ & $52: 12: 36.36$ & 23.4 & $1.99 \mathrm{E}-15$ & 2 & $\mathrm{y}$ & - & - & \\
\hline 30 & $14: 10: 57.079$ & $52: 11: 22.48$ & $14: 10: 57.27$ & $52: 11: 23.7$ & 24.5 & $9.13 \mathrm{E}-16$ & 3 & $\mathrm{n}$ & - & - & \\
\hline 31 & $14: 10: 57.168$ & $52: 11: 30.48$ & $14: 10: 57.41$ & $52: 11: 30.71$ & 21.54 & $2.76 \mathrm{E}-15$ & 2 & $\mathrm{n}$ & - & - & \\
\hline 33 & $14: 10: 58.606$ & $52: 7: 11.21$ & $14: 10: 58.79$ & $52: 7: 12.03$ & 20.52 & $3.49 \mathrm{E}-14$ & 3 & $\mathrm{n}$ & 0.394 & $1.88 \mathrm{E} 43$ & QSO \\
\hline 34 & $14: 10: 59.369$ & $52: 11: 52.94$ & 14:10:59.61 & $52: 11: 53.37$ & 22.27 & $4.84 \mathrm{E}-15$ & 2 & $\mathrm{y}$ & - & - & \\
\hline 36 & 14:11:3.894 & $52: 17: 55.07$ & $14: 11: 4.19$ & $52: 17: 55.1$ & 19.12 & $1.45 \mathrm{E}-14$ & 1 & $\mathrm{y}$ & - & - & \\
\hline 37 & 14:11:4.172 & $52: 11: 34.83$ & 14:11:4.35 & $52: 11: 35.38$ & 22.37 & $1.41 \mathrm{E}-15$ & 3 & $\mathrm{y}$ & - & - & \\
\hline 39 & 14:11:7.011 & $52: 19: 11.86$ & $14: 11: 7.3$ & 52:19:12: & 22.93 & $1.64 \mathrm{E}-15$ & 1 & $\mathrm{n}$ & - & - & \\
\hline 41 & $14: 11: 8.803$ & $52: 16: 44.4$ & 14:11:9.1 & $52: 16: 44.8$ & 22.8 & $6.03 \mathrm{E}-15$ & 1 & $\mathrm{y}$ & - & - & \\
\hline 43 & $14: 11: 10.674$ & $52: 9: 46.88$ & $14: 11: 10.56$ & $52: 9: 45.38$ & 24.5 & $1.10 \mathrm{E}-15$ & 3 & $\mathrm{n}$ & - & - & \\
\hline 44 & 14:11:13.41 & $52: 13: 40.4$ & $14: 11: 13.67$ & $52: 13: 40.96$ & 20.92 & 4.60E-15 & 1 & $\mathrm{y}$ & - & - & \\
\hline 46 & $14: 11: 14.193$ & $52: 6: 28.41$ & 14:11:14.41 & $52: 6: 28.84$ & 20.61 & 1.19E-14 & 3 & $\mathrm{y}$ & - & - & \\
\hline 47 & $14: 11: 14.296$ & $52: 16: 10.49$ & 14:11:14.61 & $52: 16: 10.82$ & 20.93 & $6.76 \mathrm{E}-15$ & 1 & $\mathrm{n}$ & 1.712 & $1.36 \mathrm{E} 44$ & QSO \\
\hline 48 & $14: 11: 17.424$ & 52:17:0.67 & $14: 11: 17.7$ & $52: 17: 2.11$ & 22.24 & $1.99 \mathrm{E}-15$ & 1 & $\mathrm{y}$ & - & - & \\
\hline 50 & $14: 11: 18.303$ & $52: 17: 18.17$ & $14: 11: 18.8$ & $52: 17: 15.81$ & 19.79 & $5.40 \mathrm{E}-16$ & 1 & $\mathrm{n}$ & 0.278 & $1.61 \mathrm{E} 42$ & Gal \\
\hline 51 & $14: 11: 19.271$ & 52:13:59.77 & 14:11:19.59 & 52:14:0.37 & 20.81 & $1.79 \mathrm{E}-14$ & 1 & $\mathrm{y}$ & - & - & \\
\hline 52 & $14: 11: 19.669$ & $52: 15: 46.07$ & 14:11:20:00 & $52: 15: 46.6$ & 23.0 & $1.78 \mathrm{E}-15$ & 1 & $\mathrm{n}$ & - & - & \\
\hline 63 & $14: 11: 20.497$ & $52: 14: 10.64$ & $14: 11: 20.79$ & $52: 14: 11.59$ & 15.07 & $1.12 \mathrm{E}-15$ & 1 & $\mathrm{n}$ & - & - & \\
\hline 65 & $14: 11: 20: 6$ & $52: 17: 14: 75$ & 14:11:20:86 & $52: 17: 15: 97$ & $19: 13$ & $7.52 \mathrm{E}-16$ & 1 & $\mathrm{n}$ & - & - & \\
\hline 69 & $14: 11: 21.218$ & 52:18:40.47 & 14:11:21.65 & $52: 18: 39.03$ & 19.32 & $9.43 \mathrm{E}-16$ & 1 & $\mathrm{n}$ & - & - & \\
\hline 73 & $14: 11: 22.199$ & 52:14:17.99 & $14: 11: 22.5$ & 52:14:18.6 & 23.9 & $4.02 \mathrm{E}-16$ & 1 & $\mathrm{y}$ & - & - & \\
\hline 74 & $14: 11: 22.574$ & $52: 16: 59.34$ & $14: 11: 22.85$ & 52:17:0.44 & 20.27 & 4.69E-16 & 1 & $\mathrm{n}$ & 0.534 & $2.23 \mathrm{E} 41$ & Gal \\
\hline 75 & $14: 11: 23.209$ & 52:13:31.04 & $14: 11: 23.52$ & $52: 13: 31.8$ & 19.66 & $5.65 \mathrm{E}-14$ & 1 & $\mathrm{n}$ & 0.474 & $4.74 \mathrm{E} 43$ & QSO \\
\hline 76 & $14: 11: 23.381$ & $52: 18: 23.62$ & $14: 11: 23.25$ & $52: 18: 20.63$ & 20.3 & $8.56 \mathrm{E}-16$ & 1 & $\mathrm{n}$ & 0.374 & 4.07E41 & QSO \\
\hline 77 & $14: 11: 23.813$ & $52: 13: 45.84$ & $14: 11: 24.12$ & $52: 13: 46.9$ & 22.56 & $5.07 \mathrm{E}-16$ & 1 & $\mathrm{n}$ & - & - & \\
\hline 79 & $14: 11: 24.503$ & $52: 19: 23.85$ & 14:11:24.75 & 52:19:23.99 & 20.83 & $6.56 \mathrm{E}-15$ & 1 & $\mathrm{y}$ & - & - & \\
\hline 83 & $14: 11: 25.935$ & 52:18:47.99 & 14:11:26.18 & 52:18:47.99 & 21.66 & $4.75 \mathrm{E}-15$ & 1 & $\mathrm{y}$ & - & - & \\
\hline 87 & $14: 11: 28.499$ & $52: 19: 29.85$ & 14:11:28.36 & 52:19:30.08 & 22.45 & $1.88 \mathrm{E}-15$ & 1 & $\mathrm{y}$ & - & - & \\
\hline 89 & $14: 11: 28.822$ & 52:14:34.37 & 14:11:28.35 & $52: 14: 33.35$ & 19.3 & $4.21 \mathrm{E}-16$ & 1 & $\mathrm{n}$ & - & - & \\
\hline 91 & $14: 11: 29.495$ & $52: 16: 39.51$ & 14:11:29.7 & $52: 16: 40.22$ & 22.42 & 4.27E-16 & 1 & $\mathrm{n}$ & - & - & \\
\hline 92 & $14: 11: 29.732$ & $52: 18: 5.26$ & $14: 11: 30.03$ & 52:18:5.79 & 22.36 & $7.96 \mathrm{E}-16$ & 1 & $\mathrm{y}$ & - & - & \\
\hline 93 & $14: 11: 30.625$ & 52:14:22.96 & 14:11:30.97 & 52:14:23.64 & 14.22 & $1.81 \mathrm{E}-15$ & 1 & $\mathrm{n}$ & 0 & - & Star \\
\hline 94 & $14: 11: 31.96$ & $52: 13: 39.14$ & $14: 11: 32.38$ & $52: 13: 41.17$ & 22.18 & $2.96 \mathrm{E}-15$ & 1 & $\mathrm{n}$ & - & - & \\
\hline 95 & $14: 11: 32.413$ & $52: 17: 54.82$ & 14:11:32.66 & $52: 17: 55.71$ & 17.56 & $6.88 \mathrm{E}-16$ & 1 & $\mathrm{n}$ & 0.159 & $1.22 \mathrm{E} 41$ & Gal \\
\hline 97 & $14: 11: 32.983$ & $52: 15: 41.54$ & 14:11:32.95 & $52: 15: 43.86$ & 23.37 & $1.23 \mathrm{E}-15$ & 1 & $\mathrm{n}$ & - & - & \\
\hline 98 & $14: 11: 33.121$ & $52: 12: 56.34$ & 14:11:33.48 & $52: 12: 57.07$ & 23.0 & $4.43 \mathrm{E}-16$ & 4 & $\mathrm{n}$ & - & - & \\
\hline 100 & $14: 11: 36.818$ & 52:18:45.47 & 14:11:36.89 & $52: 18: 46.35$ & 21.28 & $4.91 \mathrm{E}-15$ & 1 & $\mathrm{n}$ & - & - & \\
\hline 101 & $14: 11: 37.192$ & 52:18:27.08 & 14:11:37.09 & 52:18:27.9 & 21.7 & $1.81 \mathrm{E}-15$ & 1 & $\mathrm{y}$ & - & - & \\
\hline 102 & 14:11:38.83 & $52: 16: 22.37$ & 14:11:39.06 & $52: 16: 22.86$ & 21.9 & $3.21 \mathrm{E}-15$ & 1 & $\mathrm{y}$ & - & - & \\
\hline 104 & $14: 11: 42.016$ & $52: 12: 30.02$ & 14:11:41.62 & $52: 12: 29.56$ & 21.8 & $1.31 \mathrm{E}-15$ & 4 & $\mathrm{y}$ & - & - & \\
\hline 107 & 14:11:42.569 & $52: 19: 5.78$ & $14: 11: 42.7$ & $52: 19: 6.3$ & 24.09 & $8.48 \mathrm{E}-16$ & 1 & $\mathrm{y}$ & - & - & \\
\hline 108 & $14: 11: 42.912$ & $52: 16: 35.58$ & 14:11:43.1 & $52: 16: 36.0$ & 22.8 & $1.48 \mathrm{E}-15$ & 1 & $\mathrm{n}$ & - & - & \\
\hline 111 & 14:11:44.33 & 52:18:33.05 & 14:11:44.56 & $52: 18: 33.73$ & 18.73 & $7.68 \mathrm{E}-16$ & 1 & $\mathrm{n}$ & - & - & \\
\hline 113 & $14: 11: 47.561$ & 52:17:1.87 & 14:11:47.75 & $52: 17: 2.41$ & 21.25 & $1.40 \mathrm{E}-15$ & 1 & $\mathrm{n}$ & - & - & \\
\hline 114 & $14: 11: 47.976$ & $52: 17: 58.93$ & 14:11:48.2 & $52: 17: 59.62$ & 22.31 & $3.91 \mathrm{E}-15$ & 1 & $\mathrm{y}$ & - & - & \\
\hline
\end{tabular}


Table 1. continued.

\begin{tabular}{|c|c|c|c|c|c|c|c|c|c|c|c|}
\hline & \multicolumn{2}{|c|}{$\overline{\bar{X} \text { position }}$} & \multicolumn{2}{|c|}{ Optical position } & \multicolumn{2}{|r|}{$\overline{\bar{X} \text { flux (cgs) }}$} & & & & & \\
\hline$n$ & $\overline{\mathrm{RA}}$ & Dec & RA & Dec & $R$ mag & $0.5-10 \mathrm{keV}$ & Chip & Stack & $\bar{z}$ & $\overline{L X}(\mathrm{erg} / \mathrm{s})$ & Class \\
\hline 115 & $14: 11: 48.227$ & $52: 11: 29.87$ & $14: 11: 48.48$ & $52: 11: 31.01$ & 20.58 & $3.97 \mathrm{E}-15$ & 4 & $\mathrm{n}$ & - & - & \\
\hline 118 & $14: 11: 51.042$ & $52: 14: 57.16$ & $14: 11: 51.2$ & $52: 14: 57.07$ & 24.07 & $7.73 \mathrm{E}-16$ & 1 & $\mathrm{y}$ & - & - & \\
\hline 122 & $14: 11: 55.052$ & $52: 18: 22.68$ & $14: 11: 54.73$ & $52: 18: 24.25$ & 22.31 & $1.41 \mathrm{E}-15$ & 1 & $\mathrm{y}$ & - & - & \\
\hline 123 & $14: 11: 55.038$ & $52: 11: 34.12$ & $14: 11: 55.3$ & $52: 11: 35.46$ & 21.7 & $2.06 \mathrm{E}-15$ & 4 & $\mathrm{n}$ & - & - & \\
\hline 124 & $14: 11: 55.399$ & $52: 18: 2.77$ & $14: 11: 55.56$ & $52: 18: 3.48$ & 20.28 & $7.22 \mathrm{E}-15$ & 1 & $\mathrm{n}$ & 1.636 & $1.30 \mathrm{E} 44$ & QSO \\
\hline 126 & $14: 11: 56.267$ & $52: 16: 37.02$ & $14: 11: 56.3$ & $52: 16: 36.7$ & 23.44 & $7.81 \mathrm{E}-16$ & 1 & $\mathrm{n}$ & - & _- & \\
\hline 128 & $14: 11: 57.648$ & $52: 14: 10.31$ & $14: 11: 57.77$ & $52: 14: 11.59$ & 21.68 & $1.16 \mathrm{E}-15$ & 4 & $\mathrm{n}$ & - & - & \\
\hline 131 & 14:12:0.065 & $52: 13: 29.57$ & $14: 11: 59.54$ & 52:13:29.35 & 20.08 & $5.10 \mathrm{E}-15$ & 4 & $\mathrm{y}$ & - & - & \\
\hline
\end{tabular}

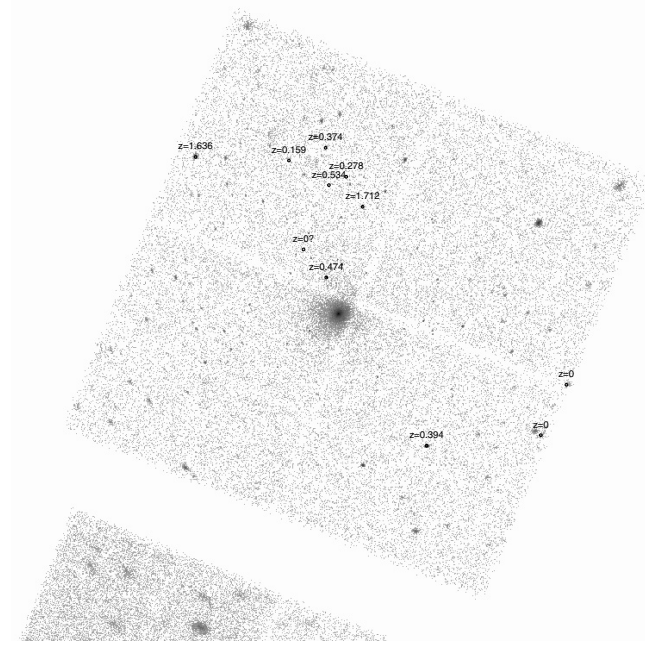

Fig. 1. The position of the spectroscopically-identified X-ray sources inside the 3C 295 field.

the exposure time and the sensitivity. This technique is particularly useful in our case, because we are looking for a number of sources at similar redshifts: individual spectral features would be significantly enhanced in the stacked spectrum, if the sources are truly at the same redshift. One of the strongest features in AGN X-ray spectra is the iron $\mathrm{k} \alpha$ line at $6.4 \mathrm{keV}$, which would be an obvious line to try to identify in a stacked spectrum of Chandra sources.

\subsection{X-ray data preparation}

Chandra observed the $16^{\prime} \times 16^{\prime}$ field around the 3C 295 cluster using ACIS-I (Garmire 1997) on May 18, 2001. The aim point of the observation was located at the position of 3C 295 $\alpha=14: 11: 10, \delta=+52: 13: 01$ (J2000) and the exposure time was $\sim 92 \mathrm{ks}$. D'Elia et al. (2005) identified 121 sources in the 0.5-10 keV band (see Fig. 3, top panel), using the PWDetect algorithm (Damiani et al. 1997a,b). We search here for the $6.4 \mathrm{keV}$ iron line emission at $z \sim 0.46$, and therefore extracted, from the original data, images in the $4.1 \div 4.9 \mathrm{keV}$ energy range. Fifty-one of the 121 sources detected in the $0.5-10 \mathrm{keV}$ band are detected in this image at a confidence level higher than $5 \sigma$. Four of these sources were spectroscopically-identified (see Table 1) to be at a redshift different from that of 3C 295 (see Fig. 3, bottom panel). We excluded these sources from the analysis described below. We were therefore left with 47 sources.

\subsection{X-ray stacked spectrum}

We extracted a composite "stacked" spectrum from source region files of radii 3 arcsec centered at the positions of these 47 sources. We used the task PSExtract of the Chandra Interactive Analysis of Observations (CIAO) software (http:// cxc.harvard.edu/ciao). A background spectrum was extracted in several source-free regions distributed close to the 47 sources. Response matrices and effective-area files were created at the centroid of the 47 sources and added together to obtain average responses.

The stacked spectrum was modeled using XSPEC version 11. We used a model that included the following components: 1) a power law with both energy index and normalization as free parameters; 2) galactic absorption toward the 3C 295 field with $N_{\mathrm{H}}$ fixed at the value of $1.33 \times 10^{20} \mathrm{~cm}^{-2}$ (Dickey \& Lockman 1990); 3) a neutral absorber at the redshift of 3C $295(z=0.46)$ with column density free to vary. The fit of this model produces a reduced $\chi^{2}$ of 1.07 for 142 degrees of freedom. The residuals, plotted in Fig. 4, show a narrow (at the resolution of the Chandra ACIS-I) line at $E \sim 4.4 \mathrm{keV}$, the energy of the iron $\mathrm{K} \alpha$ line at the redshift of the cluster. A fit to the stacked spectrum using the same model as above plus a Gaussian line at $z=0.46$ produces an improvement of the $\chi^{2}$ value to 0.96 for three additional parameters. This is significant at the $99.8 \%$ level, using the $\mathrm{F}$ test. The bottom panel of Fig. 4 shows the $\chi^{2}$ confidence contour for the normalization and energy of this line. This plot confirms a detection significant at higher than a $99.7 \%$ confidence level and constrains the energy of the line to the range $6.20 \div 6.47 \mathrm{keV}$ (90\% confidence interval), thus excluding an identification with the $6.7 \mathrm{keV}$, helium-like, iron line from the hot intra-cluster medium at higher than a $4 \sigma$ confidence level. The rest-frame equivalent width of the $6.4 \mathrm{keV} \mathrm{K} \alpha$ line is $E W=230 \pm 90 \mathrm{eV}$.

We verified that the line in the stacked spectrum was not due to the contribution of a single source. First, we excluded the type $1 \mathrm{AGN}$ at $z=0.474$ (Fig. 2) from the stacked spectrum. This did not significantly change the quality of the fits and the results reported above. Secondly, we verified that the $4.4 \mathrm{keV}$ feature was not due to a selection effect. For this purpose, we stacked the spectra of the 3 sources with an optically-identified redshift far from $z=0.46$ in the energy range $4.1 \div 4.9 \mathrm{keV}$. This spectrum does not show any sign of a feature at $4.4 \mathrm{keV}$. The $90 \%$ limit on the equivalent width of a line at $4.4 \mathrm{keV}$ is $200 \mathrm{eV}$. Thirdly, we divided the $4.1 \div 4.9 \mathrm{keV}$ sample into four groups, according to the location of the sources in the four quadrants plotted in the bottom panel of Fig. 3. We then performed a stacking analysis similar to that performed for the complete sample. We find that the feature at $\sim 4.4 \mathrm{keV}$ is visible in the $\mathrm{NE}(E W=150 \pm 100 \mathrm{eV}), \mathrm{NW}(E W=50 \pm 150 \mathrm{eV})$, and $\mathrm{SE}(E W=60 \pm 150 \mathrm{eV})$ quadrants. The feature was not detected, however, at a significance level higher than $99.7 \%$ in any 

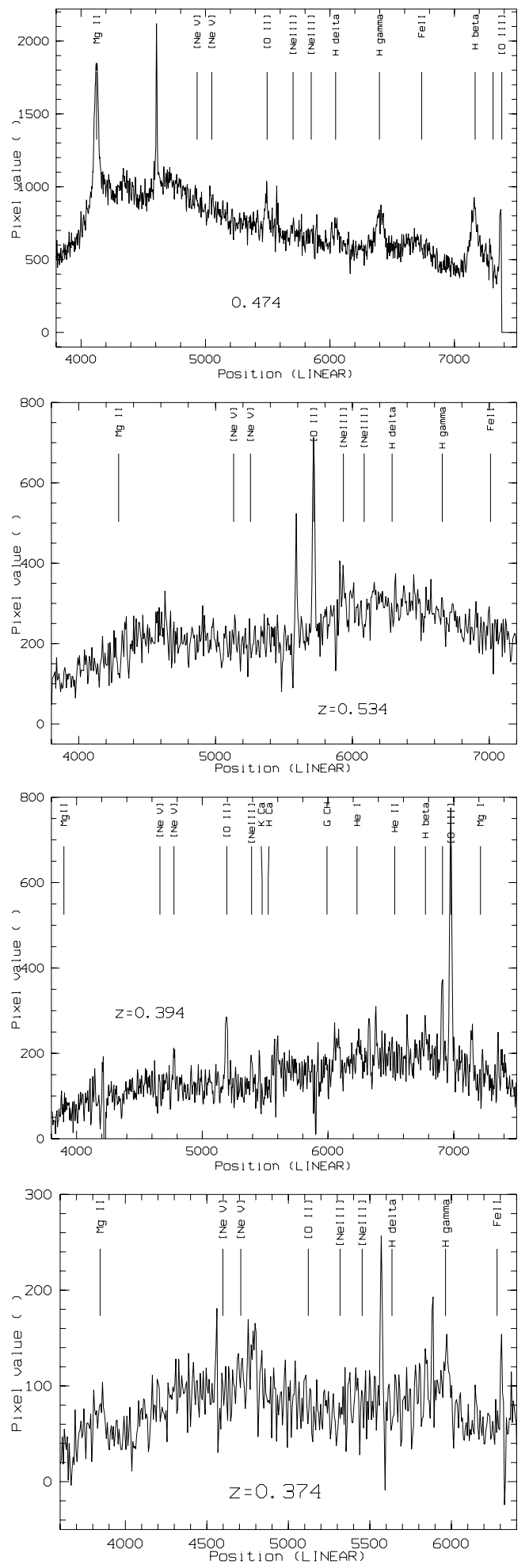

Fig. 2. The optical spectra of the 4 sources whose redshift is closer to that of the central cluster of the 3C 295 field $(z=0.46)$.

of these spectra. Although there is a hint that the iron K $\alpha$ equivalent width is higher for the NE quadrant, the quality of our data is not sufficiently high to understand whether the signal is mainly associated with sources in this quadrant or is distributed more evenly in two or three quadrants. Deeper observations are clearly required for this purpose.

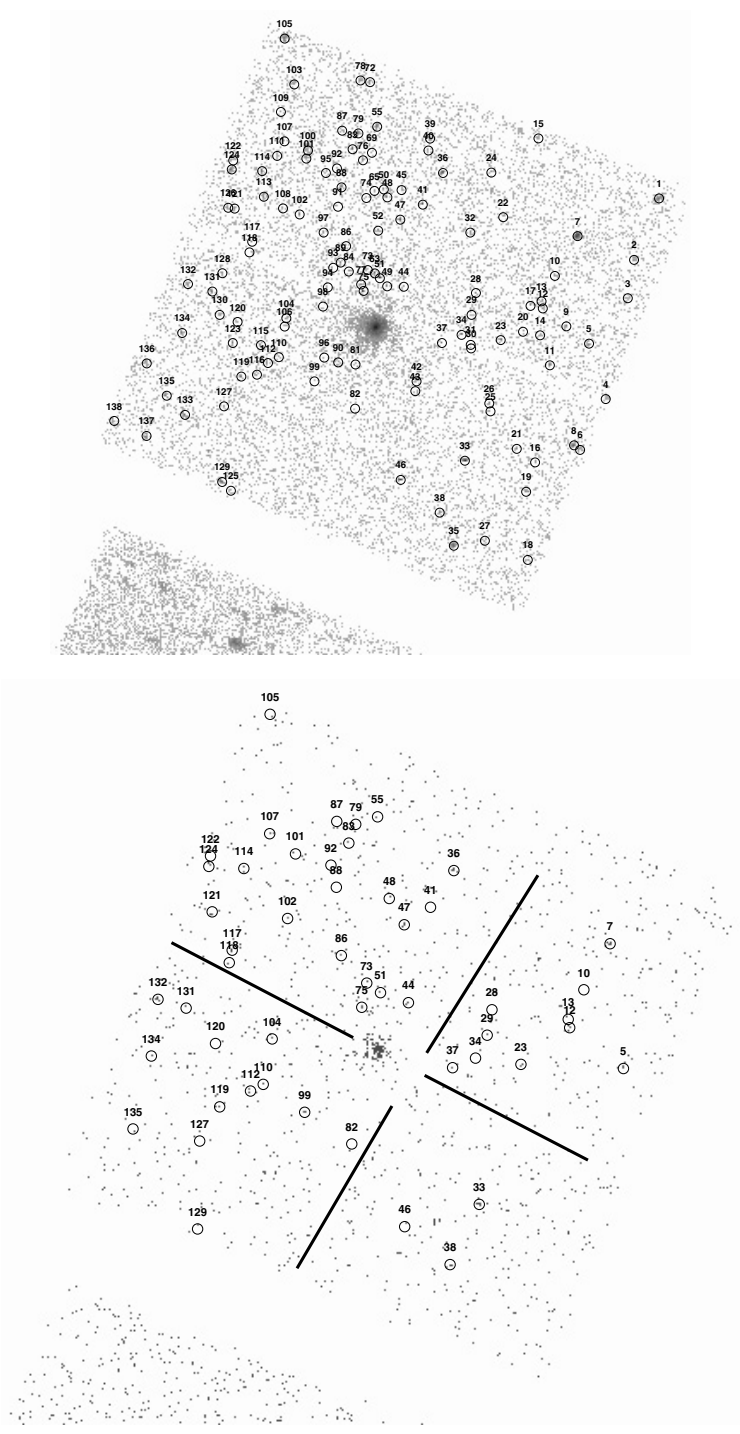

Fig. 3. Top panel: the $0.5-10 \mathrm{keV}$ Chandra image of the 3C 295 field; circles represent the 121 sources identified with the PWDetect algorithm. Bottom panel: the 4.1-4.9 keV image of the 3C 295 field; circles represent the 51 sources selected for the production of the stacked X-ray spectrum, and black lines divide the image in its four quadrants.

\section{Conclusions and discussion}

A 92 ks Chandra observation of the field around the $z=0.46$ 3C 295 cluster of galaxy shows an excess of sources visible in the NE quadrant (Fig. 3). D'Elia et al. (2005) performed a detailed study of the field, applying three different methods of analysis, namely, the $\log N-\log S$ computed separately for the four ACIS-I chips, the two-dimensional Kolmogorov-Smirnov (KS) test, and the 2 points angular correlation function. All of these analysis methods suggest an asymmetric distribution of sources in the NE region of the field and a strong clustering on scales of a few arcmins.

Cappi et al. (2001) discussed four possible causes of these "excess" sources: (1) gravitationally-lensed very faint sources; (2) rapid evolution of cluster AGNs or starburst galaxies (3) cosmic variance of background sources; (4) LSS associated with the cluster. Since the surplus sources are not symmetrically distributed around the cluster, our results rule out lensing by the cluster potential well and AGNs within the cluster. 


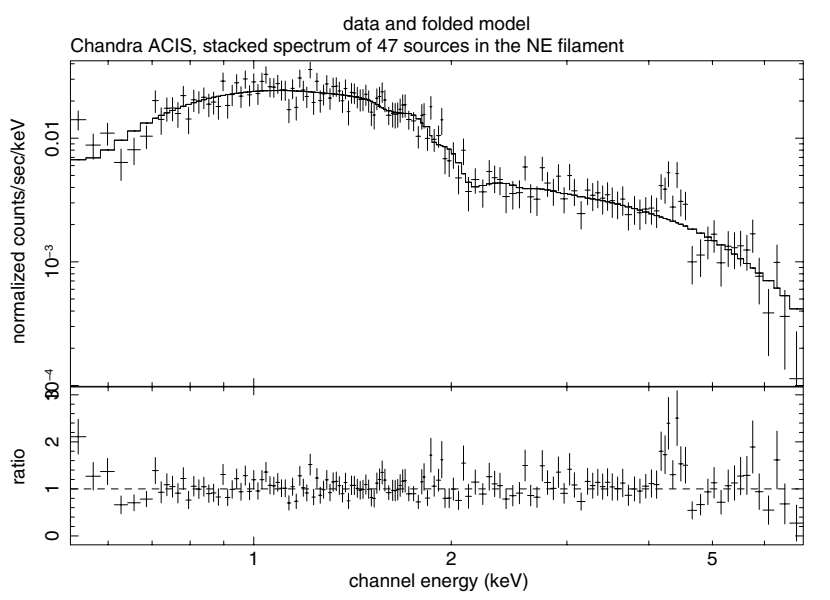

delia 5-Dec-2007 16:50

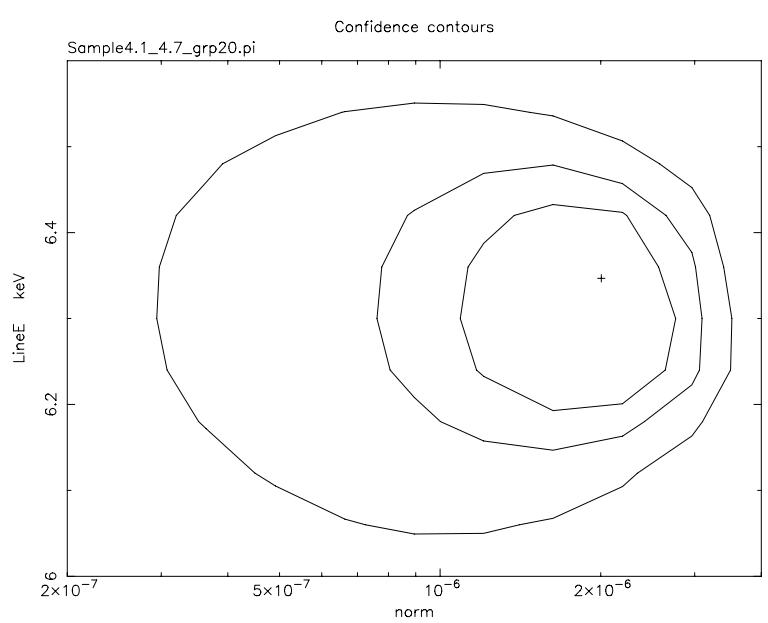

Fig. 4. Upper panel: the stacked spectrum of the $47 \mathrm{X}$-ray sources of Fig. 3 belonging to the $3 \mathrm{C} 295$ field. A redshifted Fe line at $E \sim 4.4 \mathrm{keV}$ is clearly visible. Lower panel: the confidence contours of the line energy and normalization; the rest frame energy of the line is in the range 6.2-6.47 (90\% confidence interval).

In this paper, we studied in more detail the association of the excess sources with the cluster. We used two different approaches, namely a direct spectroscopic identification of the optical counterpart of the X-ray sources, and X-ray stacked spectra.

Twenty-six out of $121 \mathrm{X}$-ray sources were identified with an optical counterpart brighter than $R=22$. Reliable redshifts were obtained for 11 sources. Three objects were stars, two of which were located in the SW quadrant of the Chandra observation, and the third was a questionable identification. One type 1 AGN has a redshift very similar to that of 3C 295 and it is located in the NE quadrant, in addition to two of the other three objects with redshifts in the range $0.37 \div 0.53$. The remaining sources were two $z>1$ QSOs and two low-redshift galaxies $(z<0.3)$. These results enhance the asymmetric distribution found by D'Elia et al. (2005), since the two stars are located in the SW, less-populated chip of the Chandra field.

Further evidence for an association of at least some of the "excess" sources with the LSS linked to 3C 295 comes from the analysis of the X-ray "stacked" spectrum of 51 Chandra sources detected in the $4.1 \div 4.9 \mathrm{keV}$ band. This band includes the redshifted iron $\mathrm{K} \alpha$ line at $z \sim 0.46$. We excluded objects with a spectroscopic redshift significantly different from that of the cluster. We detected in the stacked spectrum an emission line at $\sim 4.4 \mathrm{keV}$ with a statistical significance better than $99.7 \%$ confidence level. Assuming the redshift equal to that of the cluster, the line energy was measured to be in the range $6.20 \div$ $6.47 \mathrm{keV}$ (90\% confidence interval). This range excludes the $6.7 \mathrm{keV}$ helium-like iron line from the hot intracluser medium at better than $4 \sigma$. We verified that the line in the stacked spectrum is not due to the contribution of a single source. We conclude that the detection of the redshifted line is a strong indication that at least several of the excess sources lie at $z \sim 0.46$, and have an AGN signature. They are therefore associated with the central cluster and possibly form a "filament" connected with 3C 295.

At $z=0.46$, the $0.5-10 \mathrm{keV}$ luminosity of these sources is in the range $10^{42} \div 10^{44} \mathrm{erg} \mathrm{s}^{-1}$ (median $=3 \times 10^{42} \mathrm{erg} \mathrm{s}^{-1}$ ). These luminosities are typical for Seyfert-like galaxies. Martini et al. (2006), studying 40 sources in the field of 8 Chandra lowredshift clusters $(0.05<z<0.31)$, found that at least 35 of these objects have AGN signatures. Such signatures were discovered not only using optical emission lines, but, above all, by observing the X-ray spectral shapes and the multiwavelength flux ratio. The authors concluded that dilution of low-luminosity AGN spectral signatures by host galaxy starlight and obscuration of accretion onto the central black hole did not easily allow these sources to be optically classified as AGNs. As a consequence, the AGN fraction in clusters of galaxies is a factor of 5 higher than previously observed. This agrees with our result, since the excess sources close to 3C 295 have an AGN signature in X-rays, but most have faint optical counterparts.

The virial radius, which is the radius at which the density contrast is 2500, of 3C 295 is $r=0.4 \mathrm{Mpc}$ (Allen, Schmidt $\&$ Fabian). The integration of the density profile up to this radius, yields a mass of $2 \times 10^{14} M_{\odot}$ and a velocity dispersion of $\sim 1300 \mathrm{~km} \mathrm{~s}^{-1}$ (Dressler \& Gunn 1992). The allowed $\Delta z$ compared to these data is $\sim 0.005$. On the other hand, an association with 3C 295 defines a spatial scale of $2 \mathrm{Mpc}$ ( 5 arcmin) for the adopted cosmology; this implies that we are sampling a cosmic filament of the LSS and not cluster members. Ruderman $\&$ Ebeling (2005) analyzed the spatial distribution of a sample of 508 X-ray sources detected by Chandra ACIS-I in 51 massive galaxy clusters $(z \sim 0.3 \div 0.7)$. They found a significant $(8 \sigma)$ excess within $3.5 \mathrm{Mpc}$ from the cluster center, and they believed it to be caused by AGNs connected to the central clusters. They concluded that for relaxed clusters, such as 3C 295, two spikes in the spatial distribution of the X-ray sources emerged. The authors believed that the first scale of $<0.5 \mathrm{Mpc}$, was due to galaxy interactions involving the giant ellipticals close to the central core, while the second scale at $2-3 \mathrm{Mpc}$, was caused by galaxy mergers. These mergers are more likely to occur in a transition region between the cluster and the field, where the galaxy velocity dispersion is lower, but become rarer between the two spatial scale peaks, where the velocities are higher. The position of this second spike intriguingly agrees with our spatial scale of $2 \mathrm{Mpc}$; this can indicate that we are mapping a transition region between the cluster and the field, that is, a cosmic "filament", where pronounced AGN activity is present.

The integral of the field galaxy luminosity function at $z=$ 0.5 (e.g. Poli et al. 2001) provides $\sim 0.13$ galaxies $\mathrm{Mpc}^{-3}$ for $M_{B}<-17$, a resonable faint end optical luminosity, corresponding to our lower X-ray luminosities. If we assume that roughly one tenth of the galaxies are active $\mathrm{X}$-ray sources of $L_{\mathrm{X}}>3 \times 10^{41} \mathrm{erg} \mathrm{s}^{-1}$, then we would expect $\sim 0.013 \mathrm{X}$-ray sources $\mathrm{Mpc}^{-3}$. Since we calculate $\sim 0.9$ sources $\mathrm{Mpc}^{-3}$, this implies a galaxy overdensity of $\approx 70$, with of course a large (factor of 2-4) positive and negative uncertainty, because of the uncertainties in our space densities and assumptions. Again, this number is interestingly close to the expected galaxy overdensity of 
filaments $\sim 10 \div 10^{2}$, and much smaller than the overdensities of clusters of galaxies $\left(\sim 10^{3} \div 10^{4}\right.$, the density contrast at the virial radius being $\sim 2500$ ). This is further confirmation that we are probing a cosmic "filament" connected to the galaxy cluster 3C 295 .

Acknowledgements. V.D. acknowledges support from ASI contract I/011/07/0, $\mathrm{I} / \mathrm{R} / 039 / 04$ and $\mathrm{I} / \mathrm{R} / 023 / 05 / 0$.

\section{References}

Allen, S. W., Schmidt, R. W., \& Fabian, A. C. 2001, MNRAS, 328, L37 Almaini, O., Scott, S. E., Dunlop, J. S., et al. 2003, MNRAS, 338, 303 Best, P. N., van Dokkum, P. G., Franx, M., \& Rottgering, H. J. A. 2002, MNRAS, 330,17

Cappelluti, N., Cappi, M., Dadina, M., et al. 2005, A\&A, 430, 39

Cappi, M., Mazzotta, P., Elvis, M., et al. 2001, ApJ, 548, 624

Daddi, E., Broadhurst, T., Zamorani, G., et al. 2001, A\&A, 376, 825

Damiani, F., Maggio, A., Micela, G., \& Sciortino, S. 1997a, ApJ, 483, 350

Damiani, F., Maggio, A., Micela, G., \& Sciortino, S. 1997b, ApJ, 483, 370
Davé, R., Spergel, D. N., Steinhardt, P. J., \& Wandelt, B. D. 2001, ApJ, 547, 574 D'Elia, V., Fiore, F., Elvis, M., et al. 2004, A\&A, 422, 11

Dickey, J. M., \& Lockman, F. J. 1990, ARA\&A, 28, 215

Dressler, A., \& Gunn, J. E. 1992, ApJS, 78, 1

Fiore, F., Nicastro, F., Savaglio, S., Stella, L., \& Vietri, M. 2000, ApJ, 544, L7

Garmire, G. P. 1997, A\&AS, 29, 283

Giavalisco, M., \& Dickinson, M. 2001, ApJ, 550, 177

Martini, P., Kelson, D. D., Mulchaey, J. S., \& Trager, S. C. 2002, ApJ, 576, L109 Martini, P., Kelson, D. D., Kim, E., Mulchaey, J. S., \& Athey, A. A. 2006, ApJ, 644, 116

Mathur, S., Weinberg, D. H., \& Chen, X. 2003, ApJ, 582, 82

Molnar, S. M., Hughes, J. P., Donahue, M., \& Joy, M. 2002, ApJ, 573, L91

Nicastro, F., Zezas, A., Drake, J., et al. 2002, ApJ, 573, 157

Nicastro, F., Zezas, A., Elvis, M., et al. 2003, Nature, 421, 719

Peacock, J. A. 1999, Cosmological physics (Cambridge: Cambridge University Press)

Pentericci, L., Kurk, J. D., Carilli, C. L., et al. 2002, A\&A, 396, 109

Poli, F., Giallongo, E., Fontana, A., Cristiani, S., \& D’Odorico, S. 2001, ApJ, 551, L45

Ruderman, J. T., \& Ebeling, H. 2005, ApJ, 623, L81

Soltan, A. M., Freyberg, M. J., \& Hasinger, G. 2002, A\&A, 395, 475

Yang, Y., Mushotzky, R. F., Barger, A. J., et al. 2003, ApJ, 585, L85

Zappacosta, L., Mannucci, F., Maiolino, R., et al. 2002, A\&A, 394, 7 